ANNALES

POLONICI MATHEMATICI

$82.3(2003)$

\title{
Regularity of certain sets in $\mathbb{C}^{n}$
}

\author{
by Nguyen Quang Dieu (Hanoi)
}

\begin{abstract}
A subset $K$ of $\mathbb{C}^{n}$ is said to be regular in the sense of pluripotential theory if the pluricomplex Green function (or Siciak extremal function) $V_{K}$ is continuous in $\mathbb{C}^{n}$. We show that $K$ is regular if the intersections of $K$ with sufficiently many complex lines are regular (as subsets of $\mathbb{C}$ ). A complete characterization of regularity for Reinhardt sets is also given.
\end{abstract}

1. Introduction. Let $K$ be a subset in $\mathbb{C}^{n}$. It is called regular (in the literature also sometimes $\mathcal{L}$-regular) if the pluricomplex Green function (or Siciak extremal function) $V_{K}$ is continuous on $\mathbb{C}^{n}$. A closely related concept is that of local regularity: a point $a \in \bar{K}$ is called a local regular point of $K$ if for every closed ball $U$ centred at $a$ the function $V_{U \cap K}$ is continuous at $a$. If every point of $\bar{K}$ is a local regular point of $K$ then we say that $K$ is locally regular.

The concepts of regularity and local regularity arise naturally in classical potential theory. For instance, the solvability of the Dirichlet problem on a domain in $\mathbb{C}$ is equivalent to the regularity of the domain (see [Ra]). These concepts also play central roles in pluripotential theory, especially in their connection to approximation theory. We could mention a well known theorem of Siciak (see Theorem 8.5 in [Si]) which gives the rate of polynomial approximation of a holomorphic function on a neighbourhood of a given regular polynomially convex compact set in $\mathbb{C}^{n}$. Remarkably enough, these concepts of regularity also get involved in other seemingly unrelated problems such as separately holomorphic functions, classifying the space of germs of holomorphic functions, etc. (see e.g. [Si], [Sa] and the references given therein).

However, in general determining whether a set is regular or locally regular at a given point is rather hard. One of the most useful criterions is

2000 Mathematics Subject Classification: 32U15, 32U35.

Key words and phrases: pluricomplex Green function, Siciak extremal function, regular set, locally regular point, Reinhardt set. 
the analytic accessibility criterion due to Pleśniak (see [Kl, p. 201] or [Ce, p. 117]). As a first application of this, one can prove that the closure of a $\mathcal{C}^{1}$ smoothly bounded domain is regular ([Kl, p. 202]).

The aim of this note is to give sufficient conditions for regularity of several types of compact sets in $\mathbb{C}^{n}$. We now describe the content of the paper. In Section 2, after giving some preparatory material we present a sufficient condition for the local regularity of a point in a given set. As a consequence, we show that the boundary of a smoothly bounded domain is locally regular. In Section 3 we deal with the regularity of Reinhardt subsets of $\mathbb{C}^{n}$. The main result in this section (Theorem 3.1) characterizes locally regular Reinhardt sets in $\mathbb{C}^{n}$. The proof of course depends heavily on the geometric nature of Reinhardt sets.

In the next section we study the regularity of certain compact Hartogs sets of the form

$$
\Omega_{\varphi}(K)=\left\{(z, w) \in K \times \mathbb{C}:|w| \leq e^{-\varphi(z)}\right\},
$$

where $\varphi$ is a real-valued continuous function on $K$. It is shown in Proposition 4.1 that $\Omega_{\varphi}(K)$ is locally regular if and only if so is $K$. Basing on an earlier example of Sadullaev, we construct an example of a regular compact set $K$ and a continuous function $\varphi$ on $K$ such that $\Omega_{\varphi}(K)$ is not regular (in $\mathbb{C}^{n+1}, n \geq 2$ ). This shows in some sense that the conclusion of Proposition 4.1 is sharp. In the course of the proof, we also discover in Lemma 4.3 that polynomial convexity of $\Omega_{\varphi}(K)$ can be interpreted in terms of plurisubharmonicity of $\varphi$ on compact sets, a concept due to Poletsky (see [Po]).

In Section 5 we consider the regularity of components of a regular set in $\mathbb{C}^{n}$. Since the standard Cantor set is regular (see [Ra]) we see that a component of a regular set is not necessarily regular. However, if a regular polynomially convex set $K$ is the union of a finite number of disjoint compact sets then each of them must be regular. This is the content of Proposition 5.1. In Section 6, we consider the concept of weighted regularity and show that local regularity is equivalent to regularity with respect to every continuous weight. Interest for this comes from the recent work of Bloom and Levenberg (see $[\mathrm{BL}]$ ) on weighted pluripotential theory.

2. Basic notions and auxiliary facts. Let $\Omega$ be an open subset in $\mathbb{C}^{n}$, and let $u: \Omega \rightarrow[-\infty, \infty)$ be an upper semicontinuous function which is not identically $-\infty$ on any connected component of $\Omega$. The function $u$ is said to be plurisubharmonic if for every complex line $l$, the restriction of $u$ to each component of $l \cap \Omega$ is either subharmonic or identically $-\infty$. The set of plurisubharmonic functions on $\Omega$ is denoted by $\operatorname{PSH}(\Omega)$.

A subset $E$ in $\mathbb{C}^{n}$ is called pluripolar if for each point $a \in E$ there is a neighbourhood $V$ of $a$ and a function $\varphi \in \operatorname{PSH}(V)$ such that $\varphi \equiv-\infty$ on 
$E \cap V$. A basic theorem of Josefson states that $E$ is pluripolar if and only if there exists $u \in \operatorname{PSH}\left(\mathbb{C}^{n}\right)$ such that $\left.u\right|_{E} \equiv-\infty$.

Let $K$ be a bounded subset of $\mathbb{C}^{n}$. The pluricomplex Green function (or Siciak extremal function) of $K$ is defined as follows:

$$
V_{K}(z)=\sup \left\{u(z): u \in \mathcal{L}\left(\mathbb{C}^{n}\right), u \leq 0 \text { on } K\right\},
$$

where

$$
\mathcal{L}\left(\mathbb{C}^{n}\right)=\left\{u \in \operatorname{PSH}\left(\mathbb{C}^{n}\right): u(z) \leq \log ^{+}|z|+C\right\},
$$

the class of plurisubharmonic (psh) functions of logarithmic growth (here we use the notation $|z|=\left(\sum_{i=1}^{n}\left|z_{i}\right|^{2}\right)^{1 / 2}$ and $\left.\log ^{+}|z|=\max (0, \log |z|)\right)$. It is useful to consider the upper semicontinuous regularization of $V_{K}$,

$$
V_{K}^{*}(z):=\limsup _{\xi \rightarrow z} V_{K}(\xi)
$$

$K$ is said to be regular if $V_{K}$ is continuous on $\mathbb{C}^{n}$. Given a point $a \in \bar{K}$, we say that $a$ is a local regular point of $K$, or that $K$ is locally regular at $a$ if for every neighbourhood $U$ of $a$ the function $V_{U \cap K}$ is continuous at $a$. The set of local regular points of $K$ is denoted by $K_{\text {loc }}$.

Let $K$ be a compact subset of $\mathbb{C}^{n}$. We denote by $\widehat{K}$ the polynomial hull of $K$,

$$
\widehat{K}=\left\{\xi \in \mathbb{C}^{n}:|p(\xi)| \leq\|p\|_{K} \text { for all polynomials } p \text { in } \mathbb{C}^{n}\right\} .
$$

Given a subset $S$ of $\mathbb{C}^{n}$, a point $a \in \bar{S}$ is called thin if for every neighbourhood $U$ of $a$ and every $u \in \operatorname{PSH}(U)$ we have

$$
\limsup _{z \rightarrow a, z \in S \backslash\{a\}} u(z)<u(a) .
$$

In the one-dimensional case we have the following celebrated Wiener criterion:

WIENER'S CRITERION (Theorem 5.4.1 in [Ra]). Let $F$ be an $F_{\sigma}$ subset, and $\xi_{0} \in \mathbb{C}$. Let $\gamma \in(0,1)$, and for $n \geq 1$ define

$$
F_{n}=\left\{z \in F: \gamma^{n}<\left|z-\xi_{0}\right| \leq \gamma^{n-1}\right\} .
$$

Then $F$ is thin at $\xi_{0}$ if and only if

$$
\sum_{n \geq 1} \frac{n}{\log \left(2 / c\left(F_{n}\right)\right)}<\infty
$$

where $c\left(F_{n}\right)$ denotes the logarithmic capacity of $F_{n}$.

In higher dimensions, we do not have such a powerful tool; the analytic accessibility criterion (to be introduced shortly) however implies that $S$ is not thin at $a$ if there exists a real-analytic curve $\gamma$ such that $a \in \gamma$ and $\gamma \backslash\{a\} \subset S$. For an interesting discussion on thin sets and regular sets we refer the reader to the paper [Ce].

We now collect some useful facts from pluripotential theory: 
Proposition 2.1. Let $K$ be a bounded subset in $\mathbb{C}^{n}$.

(a) If $K$ is nonpluripolar then $V_{K}^{*}$ is plurisubharmonic on $\mathbb{C}^{n}$.

(b) $K$ is regular if and only if $V_{K}^{*} \equiv 0$ on $K ; K$ is locally regular at $a \in K$ if and only $V_{U \cap K}^{*}(a)=0$ for every neighbourhood $U$ of $a$.

(c) If $K$ is compact then $V_{K}^{*} \equiv V_{\widehat{K}}^{*}$.

(d) If $K$ is compact then for any compact set $L$ in $\mathbb{C}^{m}$ we have

$$
V_{K \times L}^{*} \equiv \max \left\{V_{K}^{*}, V_{L}^{*}\right\} .
$$

(e) For every pluripolar set $E$ in $\mathbb{C}^{n}$ we have $V_{K}^{*} \equiv V_{K \cup E}^{*}$.

(f) $K \backslash K_{\text {loc }}$ is pluripolar.

Remarks. Properties (a)-(c) follow rather easily from the definition of $V_{K}$. The more subtle ones, (d) and (e), can be found in [Si]; we will sometimes refer to (d) as the product property. Property (f) is an immediate consequence of the solution to the second problem of Lelong (Theorem 7.1 in [BT]). More precisely, let $\left\{a_{j}\right\}_{j \geq 1}$ be a countable dense subset of $K$. Then for every $k \geq 1$, by Theorem 7.1 of $[\mathrm{BT}]$ the set

$$
\left\{z \in B\left(a_{j}, 1 / k\right) \cap K: V_{B\left(a_{j}, 1 / k\right) \cap K}(z)<V_{B\left(a_{j}, 1 / k\right) \cap K}^{*}(z)\right\}
$$

is pluripolar, where $B(a, r)$ is the open ball with centre $a$ and radius $r$. Because a countable union of pluripolar sets is pluripolar, we infer from the definition of $K_{\text {loc }}$ that $K \backslash K_{\text {loc }}$ is pluripolar.

In general, it is hard to decide whether a given set $K$ is locally regular at $a \in \bar{K}$ or not. The following criterion due to Pleśniak (see [Kl, p. 201] or [Ce, p. 117]) is quite useful:

ANALYTIC ACCESSIBILITY CRITERION. Let $K$ be a subset of $\mathbb{C}^{n}$, and let $h$ be a holomorphic mapping from a neighbourhood of $[0,1]$ to $\mathbb{C}^{n}$. If $h((0,1]) \subset K_{\text {loc }}$, then $h(0) \in K_{\text {loc }}$.

Using the same idea, we give another geometric condition; of course by translation we may assume that $a=0$.

Theorem 2.2. Let $K$ be an $F_{\sigma}$ set in $\mathbb{C}^{n}(n \geq 2)$, i.e., $K$ is a countable union of closed sets, and $0 \in \bar{K}$. Assume that there exists a subset $D$ of $K$ satisfying the following conditions:

(i) For some $1 \leq k \leq n$ the set

$$
\widetilde{D}_{k}:=\left\{\left(\frac{z_{1}}{z_{k}}, \ldots, \frac{z_{k-1}}{z_{k}}, \frac{z_{k+1}}{z_{k}}, \ldots \frac{z_{n}}{z_{k}}\right):\left(z_{1}, \ldots, z_{n}\right) \in D, z_{k} \neq 0\right\}
$$

is nonpluripolar in $\mathbb{C}^{n-1}$.

(ii) For every $b \in D \backslash\{0\}$ the set $K \cap l_{b}$ (viewed as a subset of $\mathbb{C}$ ) is not thin at 0 , where $l_{b}$ denotes the complex line connecting 0 and $b$.

Then 0 is a locally regular point of $K$. 
Remark. Condition (i) is strictly weaker than requiring that $D$ is nonpluripolar. More precisely, if $D$ is nonpluripolar then for every $1 \leq k \leq n$ the set $\widetilde{D}_{k}$ is nonpluripolar in $\mathbb{C}^{n-1}$. If not then it is easy to check that $D \backslash\left\{z: z_{k}=0\right\}$ is pluripolar in $\mathbb{C}^{n}$. This implies that $D$ is pluripolar, a contradiction. On the other hand, the complex curve $\left\{\left(z, z^{2}\right): z \in \mathbb{C}\right\}$ does satisfy (i) and is clearly pluripolar.

Proof of Theorem 2.2. Let $U$ be an arbitrary closed ball centred at 0 in $\mathbb{C}^{n}$. We have to prove that $V_{U \cap K}^{*}(0)=0$. We split the proof into two steps.

STEP 1. We first show that $U \cap K$ is nonpluripolar. Assume otherwise; then there exists $\varphi \in \operatorname{PSH}\left(\mathbb{C}^{n}\right)$ such that $\varphi \equiv-\infty$ on $U \cap K$. Fix $b \in D \backslash\{0\}$. From (ii) we see that the set $K \cap U \cap l_{b}$ is $F_{\sigma}$ and not thin at 0 . So in particular it is not polar (viewed as a subset of $\mathbb{C}$ ), by Theorem 3.8.2 of [Ra]. It follows that $\varphi \equiv-\infty$ on $l_{b}$. Hence

$$
\varphi(b t)=-\infty, \quad \forall(b, t) \in(D \backslash\{0\}) \times \mathbb{C} .
$$

Since $\varphi \in \operatorname{PSH}\left(\mathbb{C}^{n}\right)$ we can find $\lambda \in \mathbb{C} \backslash\{0\}$ such that the function

$$
\psi\left(y_{1}, \ldots, y_{n-1}\right)=\varphi\left(\lambda y_{1}, \ldots, \lambda y_{k-1}, \lambda, \lambda y_{k+1}, \ldots, \lambda y_{n}\right)
$$

is plurisubharmonic on $\mathbb{C}^{n-1}$. On the other hand, (2) implies that $\psi \equiv-\infty$ on $\widetilde{D}_{k}$, which contradicts (i). Thus $U \cap K$ is nonpluripolar.

SteP 2. We will show that $V_{K \cap U}^{*}(0)=0$. Indeed, by Proposition 2.1(f) we can find $u \in \operatorname{PSH}\left(\mathbb{C}^{n}\right)$ such that

$$
K \backslash K_{\text {loc }} \subset E:=\left\{z \in \mathbb{C}^{n}: u(z)=-\infty\right\} .
$$

We claim that there exists $b \in D \backslash\{0\}$ such that $l_{b} \cap E$ is polar (viewed as a subset of $\mathbb{C}$ ). Indeed, otherwise $l_{b} \cap E$ would be nonpolar for every $b \in D \backslash\{0\}$. Therefore

$$
u(b t)=-\infty, \quad \forall t \in \mathbb{C}, \forall b \in D \backslash\{0\} .
$$

Using an argument similar to the one in Step 1 we also arrive at a contradiction to (i).

Now we fix such a point $b$ and set

$$
h(t)=V_{U \cap K}^{*}(b t) .
$$

Since $K \cap U$ is nonpluripolar, $h(t)$ is subharmonic on $\mathbb{C}$. We let

$$
S=\{t \in \mathbb{C}: b t \in U \cap K\}, \quad S^{\prime}=\{t \in \mathbb{C}: b t \in(U \cap K) \backslash E\} .
$$

It follows from (ii) and the Wiener criterion that $S$ is an $F_{\sigma}$ set satisfying (1). As the $F_{\sigma}$ set $S^{\prime}$ differs from $S$ only in a polar set we deduce that $S^{\prime}$ also satisfies (1). So by the Wiener criterion $S^{\prime}$ is not thin at 0 . Notice that from the definition of local regularity we get $h(t)=0$ for every $t \in S^{\prime}$. By putting all these facts together we obtain $h(0)=0$ or equivalently $V_{K \cap U}^{*}(0)=0$. 
Corollary 2.3. Let $D$ be a bounded domain in $\mathbb{C}^{n}$ with $\mathcal{C}^{1}$ boundary. Then $\partial D$ is locally regular.

Proof. Let $a \in \partial D$. We have to prove that $\bar{D}$ is locally regular at $a$. After a linear change of coordinates we may assume that $a=0$ and there exists a neighbourhood $U$ of $a$ in $\mathbb{C}^{n}$ and a function $\varrho \in \mathcal{C}^{1}$ satisfying

(i) $D \cap U=\{z \in U: \varrho(z)<0\}$.

(ii) $\varrho(z)=\operatorname{Re}\left(z_{1}\right)+o(|z|)$.

It follows that for each $b=\left(b_{1}, \ldots, b_{n}\right) \in D \cap U$ with $b_{1} \neq 0$ the intersection $l_{b} \cap D$ can be described near 0 by the equation $\operatorname{Re}\left(t b_{1}\right)+o(t)=0$. The implicit function theorem implies that near 0 , this intersection is a $\mathcal{C}^{1}$ curve passing through 0 . This means that conditions (i) and (ii) of Theorem 2.2 are satisfied, and completes the proof.

3. Regularity of certain Reinhardt compact sets. We call a subset $K$ in $\mathbb{C}^{n}$ a Reinhardt set if for every $\left(\theta_{1}, \ldots, \theta_{n}\right) \in \mathbb{R}^{n}$ we have

$$
\left(z_{1}, \ldots, z_{n}\right) \in K \Rightarrow\left(e^{i \theta_{1}} z_{1}, \ldots, e^{i \theta_{n}} z_{n}\right) \in K .
$$

The main result in this section gives a necessary and sufficient condition for a Reinhardt set to be locally regular. This result is slightly more general than a previous one that appeared in an earlier version of the paper. We would like to thank the referee for suggesting this generalization and an essential idea of the proof.

Theorem 3.1. Let $K$ be a Reinhardt set in $\mathbb{C}^{n}$. Put $V:=\left\{\left(z_{1}, \ldots, z_{n}\right)\right.$ : $\left.z_{1} \ldots z_{n}=0\right\}$. Then $K$ is locally regular if and only if $K \backslash V$ is dense in $K$.

Proof. If $K \backslash V$ is not dense in $K$ then we can find $a \in K \backslash \overline{K \backslash V}$. It follows that for some $r>0$ the set $B(a, r) \cap K$ is pluripolar (being contained in $V$ ). Thus $K$ cannot be locally regular at $a$. For the converse, we fix $a=\left(a_{1}, \ldots, a_{n}\right) \in \bar{K}$ and $r>0$. Set

$$
P_{r}(a):=\left\{\left(z_{1}, \ldots, z_{n}\right):\left|z_{j}-a_{j}\right|<r, \forall 1 \leq j \leq n\right\} .
$$

We have to prove that $V_{K \cap P_{r}(a)}^{*}(a)=0$. There are two cases to be considered.

CASE 1: $a=0$. Since $K \backslash V$ is dense in $K$, we can choose $\left(a_{1}^{\prime}, \ldots, a_{n}^{\prime}\right) \in$ $K \backslash V$ such that $\left|a_{j}^{\prime}\right|<r, \forall 1 \leq j \leq n$. Then

$$
\left\{z:\left|z_{j}\right|=\left|a_{j}^{\prime}\right|, \forall 1 \leq j \leq n\right\} \subset K \cap P_{r}(a) .
$$

By the product formula we infer

$$
V_{K \cap P_{r}(a)}^{*}(a) \leq \max _{1 \leq j \leq n} \log ^{+}\left|\frac{a_{j}}{a_{j}^{\prime}}\right|=0 .
$$

CASE 2: $a \neq 0$. Since $K$ is Reinhardt we may assume that $a=(0, \ldots, 0$, $\left.a_{k+1}, \ldots, a_{n}\right)$, where $0 \leq k \leq n-1$ and $a_{j}>0$ for $k+1 \leq j \leq n$. As $K \backslash V$ 
is dense in $K$ we can find $N>1 / r$ and sequences $\left\{\left(a_{1, m}, \ldots, a_{n, m}\right)\right\}_{m \geq 1} \subset$ $K \backslash V$ such that for all $m \geq 1$ we have

$$
\left|a_{j, m}\right|<\frac{1}{N+m}, \forall 1 \leq j \leq k ; \quad\left|a_{j, m}-a_{j}\right|<\frac{1}{N+m}, \forall k+1 \leq j \leq n .
$$

Now we set

$$
S_{j, m}:= \begin{cases}\left\{z_{j}:\left|z_{j}\right|=\left|a_{j, m}\right|\right\}, & 1 \leq j \leq k \\ \left\{z_{j}:\left|z_{j}\right|=\left|a_{j, m}\right|,\left|z_{j}-a_{j}\right|<r\right\}, & k+1 \leq j \leq n .\end{cases}
$$

Then

$$
\prod_{j=1}^{m} S_{j, m} \subset K \cap P_{r}(a) .
$$

Using the product formula we obtain

$$
V_{K \cap P_{r}(a)}^{*}(a) \leq \max _{1 \leq j \leq n} V_{S_{j, m}}^{*}\left(a_{j}\right)=\max _{k+1 \leq j \leq n} V_{S_{j, m}}^{*}\left(a_{j}\right), \quad \forall m .
$$

Fix $k+1 \leq j \leq n$, and put

$$
S_{j}:=\left\{z_{j}:\left|z_{j}\right|=a_{j},\left|z_{j}-a_{j}\right|<r\right\} .
$$

Then we can find $\alpha>0$ small enough and $m_{0}$ large enough such that

$$
a_{j} \in \widetilde{S}_{j}:=\left\{a_{j} e^{i t}:-\alpha \leq t \leq \alpha\right\} \subset S_{j},
$$

and

$$
\widetilde{S}_{j, m}=\left\{\left|a_{j, m}\right| e^{i t}:-\alpha \leq t \leq \alpha\right\} \subset S_{j, m}, \quad \forall m \geq m_{0} .
$$

Since the linear map $x \mapsto\left(\left|a_{j, m}\right| / a_{j}\right) x$ sends $\widetilde{S}_{j}$ onto $\widetilde{S}_{j, m}$ we obtain

$$
V_{\widetilde{S}_{j, m}}^{*}\left(\frac{\left|a_{j, m}\right|}{a_{j}} x\right) \equiv V_{\widetilde{S}_{j}}^{*}(x), \quad \forall x \in \mathbb{C} .
$$

Hence

$$
V_{S_{j, m}}^{*}\left(a_{j}\right) \leq V_{\widetilde{S}_{j, m}}^{*}\left(a_{j}\right)=V_{\widetilde{S}_{j}}^{*}\left(\frac{a_{j}^{2}}{\left|a_{j, m}\right|}\right), \quad \forall m \geq m_{0} .
$$

By letting $m$ tend to $\infty$ and taking into account the fact that $a_{j} \in \widetilde{S}_{j}$ and $\widetilde{S}_{j}$ is regular we get

$$
\lim _{m \rightarrow \infty} V_{S_{j, m}}^{*}\left(a_{j}\right)=0
$$

Combining this and (3) we obtain $V_{K \cap P_{r}(a)}^{*}(a)=0$.

4. Regularity of compact Hartogs sets. In this section we deal with regularity of compact Hartogs sets of the form

$$
\Omega_{\varphi}(K)=\left\{(z, w) \in K \times \mathbb{C}:|w| \leq e^{-\varphi(z)}\right\},
$$

where $K$ is a compact set in $\mathbb{C}^{n}$ and $\varphi \in \mathcal{C}(K)$, the set of real-valued continuous functions on $K$.

The first result in this section is the following. 
Proposition 4.1. Let $K$ be a compact set in $\mathbb{C}^{n}$ and $\varphi \in \mathcal{C}(K)$.

(a) If $\Omega_{\varphi}(K)$ is regular then $K$ is regular. in $\mathbb{C}^{n}$.

(b) $\Omega_{\varphi}(K)$ is locally regular in $\mathbb{C}^{n+1}$ if and only if $K$ is locally regular

Proof. (a) Let $m=\inf _{z \in K} \varphi(z)$. Then

$$
\Omega_{\varphi}(K) \subset K \times\left\{w:|w| \leq e^{-m}\right\} .
$$

By applying the product property we obtain

$$
\max \left\{V_{K}^{*}(z), V_{\left\{w:|w| \leq e^{-m}\right\}}^{*}(w)\right\} \leq V_{\Omega_{\varphi}(K)}^{*}(z, w), \quad \forall(z, w) \in \mathbb{C}^{n} \times \mathbb{C} .
$$

As $\Omega_{\varphi}(K)$ is regular, so is $K$ by Proposition 2.1(a).

(b) First assume that $K$ is locally regular. Take a point $p=\left(z_{0}, w_{0}\right) \in$ $\Omega_{\varphi}(K)$. Let $U$ be an arbitrary closed ball centred at $p$ in $\mathbb{C}^{n+1}$. Then we can find a closed ball $\widetilde{U}$ centred at $z_{0}$ in $\mathbb{C}^{n}$ so small that

$$
(\widetilde{U} \cap K) \times\left\{w:|w| \leq e^{-M}\right\} \subset \bar{U} \cap \Omega_{\varphi}(K),
$$

where $M=\sup _{z \in \widetilde{U} \cap K} \varphi(z)$. It follows from the product property that

$$
\begin{aligned}
V_{U \cap \Omega_{\varphi}(K)}^{*}(p) & \leq V_{(\widetilde{U} \cap K) \times\left\{|w| \leq e^{-M}\right\}}^{*}(p)=\max \left(V_{\widetilde{U} \cap K}^{*}\left(z_{0}\right), V_{\left\{|w| \leq e^{-M}\right\}}^{*}\left(w_{0}\right)\right) \\
& =\log ^{+}\left(\frac{\left|w_{0}\right|}{e^{-M}}\right) \leq M-\varphi\left(z_{0}\right) .
\end{aligned}
$$

As $\varphi \in \mathcal{C}(K)$ we deduce that $M-\varphi\left(z_{0}\right) \rightarrow 0$ when $\widetilde{U}$ shrinks to $z_{0}$. Thus for every neighbourhood $U$ of $p$ we have $V_{U \cap \Omega_{\varphi}(K)}^{*}(p)=0$. This means that $p$ is a local regular point of $\Omega_{\varphi}(K)$.

Conversely, assume that $\Omega_{\varphi}(K)$ is locally regular. Let $z_{0}$ be an arbitrary point of $K$ and $W$ be any neighbourhood of $z_{0}$ in $\mathbb{C}^{n}$. Choose $w_{0} \in \mathbb{C}$ such that $\left(z_{0}, w_{0}\right) \in \Omega_{\varphi}(K)$. Then for some constant $m$ small enough the set $W \times\left\{w:|w|<e^{-m}\right\}$ is a neighbourhood of $\left(z_{0}, w_{0}\right)$. Since $\left(z_{0}, w_{0}\right)$ is a local regular point of $\Omega_{\varphi}(K)$, by using the product property again we conclude as before that $V_{W \cap K}^{*}\left(z_{0}\right)=0$. Thus $K$ is locally regular.

The example below shows that the converse to (a) is false, or equivalently the hypothesis that $K$ is locally regular in (b) cannot be weakened to being regular.

Proposition 4.2. There exists a regular, polynomially convex set $K$ in $\mathbb{C}^{2}$ and a continuous subharmonic function $\varphi$ on $\mathbb{C}^{2}$ such that $\Omega_{\varphi}(K)$ is polynomially convex but not regular in $\mathbb{C}^{3}$.

REMARK. Since every regular polynomially convex compact set in $\mathbb{C}$ is locally regular ([Sa, p. 75]), the conclusion of Proposition 4.2 cannot hold if we take for $K$ a compact set of $\mathbb{C}$. 
For the proof of Proposition 4.2 we need the following lemma which might be of independent interest. Its proof will be given in the appendix. Before formulating it, we recall the following concept due to Poletsky (see $[\mathrm{Po}]$ ). Let $X$ be a compact subset of $\mathbb{C}^{n}$. An upper semicontinuous function $f$ on $X$ is called plurisubharmonic (written $f \in \operatorname{PSH}(X)$ ) if it is the limit of an increasing sequence of plurisubharmonic functions defined on neighborhoods of $X$.

Lemma 4.3. Let $K$ be a compact set in $\mathbb{C}^{n}$ and $\varphi \in \mathcal{C}(K)$. Then $\Omega_{\varphi}(K)$ is polynomially convex if and only if $K$ is polynomially convex and $\varphi \in$ $\operatorname{PSH}(K)$.

Proof of Proposition 4.2. We let $K$ be the compact set constructed in Proposition 8.1 of [Sa]. More precisely, $K=K_{1} \cup K_{2}$, where

$$
\begin{aligned}
& K_{1}=\left\{(z, w) \in \mathbb{C}^{2}:|z| \leq 1, w=0\right\} \\
& K_{2}=\left\{(z, w) \in \mathbb{C}^{2}:\right. z=e^{i \theta}, \operatorname{Re} w=0, \\
&\left.0 \leq \operatorname{Im} w \leq e^{1 /(\cos \theta-1)}, \theta \in[-\pi, \pi]\right\}
\end{aligned}
$$

It is proved there that $K$ is a polynomially convex, regular compact set in $\mathbb{C}^{2}$. Moreover, $K_{2} \subset K_{\text {loc }}$. Now we will show that for an appropriately chosen $\varphi$, the compact set $\Omega_{\varphi}(K)$ will have the desired properties. For this, we let $v$ be an arbitrary continuous subharmonic function on $\mathbb{C}$ satisfying

$$
v(z)=0, \quad \forall z \in \partial \Delta, \quad v \not \equiv 0 \quad \text { on } \Delta,
$$

where $\Delta$ is the open unit disk in $\mathbb{C}$. Let $\varphi(z, w)=v(z)+|w|$. Since $\varphi$ is continuous and plurisubharmonic on $\mathbb{C}^{2}$, by Lemma 4.3 the compact set $\Omega_{\varphi}(K)$ is polynomially convex in $\mathbb{C}^{3}$.

It remains to show that $\Omega_{\varphi}(K)$ is not regular. Indeed, otherwise

$$
V_{\Omega_{\varphi}(K)}^{*}(z)=0, \quad \forall z \in \Omega_{\varphi}(K) .
$$

Write

$$
\Omega_{\varphi}(K)=K_{1}^{\prime} \cup K_{2}^{\prime},
$$

where $K_{i}^{\prime}=\Omega_{\varphi}\left(K_{i}\right), i=1,2$. Since $K_{2}$ is locally regular, so is $K_{2}^{\prime}$ by Proposition 4.1(b). Now we have

$$
V_{K_{1}^{\prime} \cup K_{2}^{\prime}}^{*} \equiv V_{K_{1}^{\prime} \cup K_{2}^{\prime}}^{*} \leq V_{\widehat{K_{2}^{\prime}} \cup K_{1}^{\prime}}^{*} \leq V_{K_{1}^{\prime} \cup K_{2}^{\prime}}^{*} .
$$

Notice that $K_{1}^{\prime}$ is pluripolar in $\mathbb{C}^{3}$, so by Proposition $2.1(\mathrm{e})$ we obtain

$$
V_{K_{1}^{\prime} \cup K_{2}^{\prime}}^{*} \equiv V_{\widehat{K_{2}^{\prime}} \cup K_{1}^{\prime}}^{*}=V_{\widehat{K_{2}^{\prime}}}^{*} \text {. }
$$

Thus

$$
V_{K_{1}^{\prime} \cup K_{2}^{\prime}}^{*} \equiv V_{K_{2}^{\prime}}^{*}
$$


We deduce from (4) and (5) that $K_{1}^{\prime} \subset \widehat{K_{2}^{\prime}}$. On the other hand, by the choice of $v$ we have

$$
\begin{aligned}
& K_{1}^{\prime}=\left\{(z, w, u):(z, w) \in K_{1},|u| \leq e^{-v(z)}\right\} \\
& K_{2}^{\prime}=\left\{(z, w, u):(z, w) \in K_{2},|u| \leq e^{-|w|}\right\} \subset K_{2} \times \bar{\Delta} .
\end{aligned}
$$

It follows that $K_{1}^{\prime} \subset \mathbb{C}^{2} \times \bar{\Delta}$. By the maximum principle, $v \equiv 0$ on $\Delta$, a contradiction to the choice of $v$.

5. Regularity of components of regular compact sets. In this section, we are concerned with the regularity of components of a regular compact set $K$ in $\mathbb{C}^{n}$. It should be noticed that the standard Cantor set is nonpolar but totally disconnected. This shows that a component of a regular polynomially convex set may very well be irregular. On the other hand, it is clear that the union of two regular compact sets is again regular. The result below is a converse to this statement.

Proposition 5.1. Let $K_{1}, \ldots, K_{m}$ be disjoint compact subsets of $\mathbb{C}^{n}$. Assume $K_{1} \cup \ldots \cup K_{m}$ is regular and polynomially convex. Then $K_{1}, \ldots, K_{m}$ are regular and polynomially convex.

Proof. It is clear that we need only consider the case $m=2$. Further, by symmetry it suffices to show that $K_{2}$ is regular and polynomially convex. Let $f$ be a function equal to 0 on a neighbourhood of $K_{1}$ and to 1 on a neighbourhood of $K_{2}$. Thus $f$ is holomorphic on a neighbourhood of the polynomially convex compact set $K_{1} \cup K_{2}$. According to Theorem 8.5 of [Si], $f$ can be approximated uniformly on $K_{1} \cup K_{2}$ by a sequence $\left\{p_{n}\right\}$ of polynomials satisfying $\operatorname{deg} p_{n} \leq n$ and

$$
\left\|p_{n}\right\|_{K_{1}}^{1 / n}<\alpha, \quad\left\|p_{n}-1\right\|_{K_{2}}^{1 / n}<\alpha
$$

for some $\alpha \in(0,1)$. This implies that $p_{n}\left(K_{1}\right) \cap p_{n}\left(K_{2}\right)=\emptyset$ for $n$ sufficiently large. It follows that $p_{n}\left(\widehat{K}_{2}\right) \cap p_{n}\left(K_{1}\right)=\emptyset$, in particular $\widehat{K}_{2} \cap K_{1}=\emptyset$. Since $K_{1} \cup K_{2}$ is polynomially convex we have $\widehat{K}_{2}=K_{2}$.

Now we show that $K_{2}$ is not pluripolar. Otherwise, we would have $V_{K_{1} \cup K_{2}}^{*}=V_{K_{1}}^{*}$. Since $V_{K_{1}}^{*}(z)>0$ for all $z \in K_{2}$, we get a contradiction to the regularity of $K_{1} \cup K_{2}$. Since $K_{2}$ is not pluripolar in $\mathbb{C}^{n}$, it follows that $V_{K_{2}}^{*}$ is a plurisubharmonic function on $\mathbb{C}^{n}$.

It remains to prove that $V_{K_{2}}^{*} \equiv 0$ on $K_{2}$. For this, we apply to $(6)$ the inequality $\log (1+t)<t$ for $t>0$ obtain

$$
\frac{\log \left\|p_{n}\right\|_{K_{1}}}{n}<\log \alpha, \quad \frac{\log \left\|p_{n}\right\|_{K_{2}}}{n}<\frac{\alpha^{n}}{n} .
$$

Now we let $u$ be an arbitrary function in $\mathcal{L}\left(\mathbb{C}^{n}\right)$ with $u \leq 0$ on $K_{2}$. Choose 
$\varepsilon>0$ so small that

$$
\varepsilon\left\|V_{K_{2}}^{*}\right\|_{K_{1}}+\log \alpha<0
$$

We define

$$
v_{n}(z)=\frac{1}{1+\varepsilon}\left(\varepsilon u+\frac{\log \left|p_{n}(z)\right|}{n}-\frac{\alpha^{n}}{n}\right) .
$$

Since deg $p_{n} \leq n$ we have $v_{n} \in \mathcal{L}\left(\mathbb{C}^{n}\right)$. Further from $(7)$ and (8) we infer that $v_{n} \leq 0$ on $K_{1} \cup K_{2}$. Thus

$$
v_{n} \leq V_{K_{1} \cup K_{2}}
$$

Hence

$$
\frac{\varepsilon}{1+\varepsilon} u \leq V_{K_{1} \cup K_{2}}-\frac{1}{1+\varepsilon}\left(\frac{\log \left|p_{n}\right|}{n}-\frac{\alpha^{n}}{n}\right) .
$$

It follows that

$$
\frac{\varepsilon}{1+\varepsilon} V_{K_{2}}^{*} \leq V_{K_{1} \cup K_{2}}^{*}-\frac{1}{1+\varepsilon}\left(\frac{\log \left|p_{n}\right|}{n}-\frac{\alpha^{n}}{n}\right) .
$$

As $K_{1} \cup K_{2}$ is regular, we obtain

$$
\varepsilon V_{K_{2}}^{*}(z) \leq-\frac{\log \left|p_{n}(z)\right|}{n}+\frac{\alpha^{n}}{n}, \quad \forall z \in K_{2} .
$$

By letting $n$ go to $\infty$ we have

$$
V_{K_{2}}^{*}(z)=0, \quad \forall z \in K_{2} .
$$

Thus $K_{2}$ is regular. The proof is complete.

RemARKS. (a) The assumption that $K_{1} \cup K_{2}$ is polynomially convex cannot be entirely omitted. Indeed, it suffices to take for $K_{1}$ the circle $|z|=1$ and for $K_{2}$ the origin in $\mathbb{C}$.

(b) It would be interesting to have a more direct proof of Proposition 5.1 that does not appeal to Theorem 8.5 of [Si].

6. Weighted regularity. Let $K$ be a subset in $\mathbb{C}^{n}$ and $q: K \rightarrow$ $[-\infty, \infty)$. As in $[\mathrm{Si}]$, for each $z \in \mathbb{C}^{n}$ we define

$$
V_{K, q}(z)=\sup \left\{u(z): u \in \mathcal{L}\left(\mathbb{C}^{n}\right), u \leq q \text { on } K\right\} .
$$

We say that $K$ is regular with respect to the weight $q$ if $V_{K, q}$ is continuous on $\mathbb{C}^{n}$. It is proved in [Si] that if $K$ is locally regular then $K$ is regular with respect to every continuous weight function. In this section we will prove the converse.

Proposition 6.1. Let $K$ be a compact subset of $\mathbb{C}^{n}$. If $V_{K, q}$ is continuous on $\mathbb{C}^{n}$ for every $q \in \mathcal{C}(K)$ then $K$ is locally regular. 
Proof. Let $a \in K$, and let $U$ be any open ball centred at $a$. We have to prove that

$$
V_{K \cap U}^{*}(a)=0 .
$$

We claim first that $K \cap U$ is not pluripolar. Indeed, assume otherwise. Then by Proposition 3.11 of [Si] we obtain

$$
V_{K, q}^{*} \equiv V_{K \backslash U, q}^{*}
$$

for every $q \in \mathcal{C}(K)$. We define the following function on the compact set $(K \backslash U) \cup\{a\}$ :

$$
\widetilde{q}(z)= \begin{cases}0, & z \in K \backslash U \\ V_{K \backslash U}^{*}(a)-1, & z=a\end{cases}
$$

Let $q$ be a continuous extension of $\widetilde{q}$ to $K$. By applying (9) to $q$ we get

$$
V_{K, q}(a)=V_{K, q}^{*}(a)=V_{K \backslash U, q}^{*}(a)=q(a)+1 .
$$

This is a contradiction. Thus $K \cap U$ is not pluripolar.

Therefore we may define the following function:

$$
q(z)= \begin{cases}0, & z \in K \cap U, \\ V_{K \cap U}^{*}(z), & z \in K \backslash U .\end{cases}
$$

It is easy to check that $q$ is upper semicontinuous on $K$. Thus we can find a sequence $\left\{q_{n}\right\}_{n \geq 1}$ of continuous functions on $K$ decreasing to $q$ on $K$. Let $u$ be a function in $\mathcal{L}\left(\mathbb{C}^{n}\right)$ with $u \leq 0$ on $K \cap U$. It is clear that

$$
u(z) \leq V_{K \cap U}^{*}(z)=q(z) \leq q_{n}(z), \quad z \in K \backslash U .
$$

It follows that

$$
V_{K \cap U}(z) \leq V_{K, q_{n}}(z), \quad z \in \mathbb{C}^{n} .
$$

Since $V_{K, q_{n}}$ is continuous on $\mathbb{C}^{n}$, we obtain

$$
V_{K \cap U}^{*}(z) \leq V_{K, q_{n}}(z), \quad z \in \mathbb{C}^{n} .
$$

In particular, we have $V_{K \cap U}^{*}(a) \leq V_{K, q_{n}}(a) \leq q_{n}(a)$. Finally, by letting $n$ tend to $\infty$ we get $V_{K \cap U}^{*}(a)=0$.

\section{Appendix}

Proof of Lemma 4.3. Assume that $\varphi \in \operatorname{PSH}(K)$ and $\widehat{K}=K$. We must show that $\Omega_{\varphi}(K)$ is polynomially convex. Since $\widehat{K}=K$ we can choose a sequence $\left\{U_{n}\right\}_{n \geq 1}$ of polynomial polyhedrons decreasing to $K$ and an increasing sequence $\left\{\varphi_{n}\right\}_{n \geq 1}$ of plurisubharmonic functions on $U_{n}$ such that

$$
\lim _{n \rightarrow \infty} \varphi_{n}(z)=\varphi(z), \quad z \in K .
$$

Now for each $n \geq 1$ we define $V_{n}=U_{n} \times \mathbb{C}$ and

$$
\psi_{n}(z, w)=\log |w|+\varphi_{n}(z), \quad(z, w) \in U_{n} \times \mathbb{C} .
$$


It is clear that $V_{n}$ is Runge in $\mathbb{C}^{n+1}$ and $\psi_{n} \in \operatorname{PSH}\left(V_{n}\right)$. Furthermore

$$
\psi_{n}(z, w) \leq \log |w|+\varphi(z) \leq 0, \quad(z, w) \in \Omega_{\varphi}(K) .
$$

Since $\Omega_{\varphi}(K)$ is a compact subset of $V_{n}$, from Theorem 4.3 .3 of [Hö] we deduce that the polynomial convex hull of $\Omega_{\varphi}(K)$ equals its holomorphic convex hull in $V_{n}$. The latter, according to Theorem 4.3.4 of [Hö], is the same as the plurisubharmonic hull of $\Omega_{\varphi}(K)$ in $V_{n}$. Thus it follows from (10) that

$$
\psi_{n}(z, w) \leq 0, \quad(z, w) \in \widehat{\Omega_{\varphi}(K)} .
$$

By letting $n$ go to $\infty$ we see that $\Omega_{\varphi}(K)$ is polynomially convex.

Conversely, assume that $\Omega_{\varphi}(K)$ is polynomially convex. Since $K \times\{0\} \subset$ $\Omega_{\varphi}(K)$, we infer that $K$ is polynomially convex. It remains to prove that $\varphi \in \operatorname{PSH}(K)$. To see this, we extend $\varphi$ to a continuous function (still denoted by $\varphi$ ) on $\mathbb{C}^{n}$. Let $\left\{U_{n}\right\}$ be a sequence of Runge domains decreasing to $K$. It follows that the sequence $\left\{W_{n}\right\}_{n \geq 1}$, where

$$
W_{n}=\left\{(z, w): z \in U_{n}, \log |w|+\varphi(z)<0\right\},
$$

forms a neighbourhood basis for $K$. On the other hand, as $K$ is polynomially convex, the sequence $\left\{\widehat{W}_{n}\right\}_{n \geq 1}$ is a Stein neighbourhood basis for $K$, where $\widehat{W}_{n}$ is the (single sheeted) envelope of holomorphy of $W_{n}$. Since

$$
W_{n}=\left\{(z, w): z \in U_{n}, \log |w|+g_{n}(z)<0\right\},
$$

where $g_{n}$ is the largest plurisubharmonic minorant of $\varphi$ on $U_{n}$, the functions $g_{n}$ must increase to $\varphi$ on $K$.

The proof is thereby finished.

Acknowledgements. We are grateful to the referee for his/her accurate remarks and comments. This work was supported by the National Basic Research Program in Natural Sciences, Vietnam.

\section{References}

[BT] E. Bedford and B. Taylor, A new capacity for plurisubharmonic functions, Acta Math. 149 (1982), 1-40.

[BL] T. Bloom and N. Levenberg, Weighted pluripotential theory, preprint.

[Ce] U. Cegrell, Thin sets in $\mathbb{C}^{n}$, Univ. Iagell. Acta Math. 25 (1985), 115-120.

[Hö] L. Hörmander, An Introduction to Complex Analysis in Several Variables, 3rd ed., North-Holland, 1991.

[Kl] M. Klimek, Pluripotential Theory, Oxford Univ. Press, 1991.

[Po] E. Poletsky, Analytic geometry on compacta in $\mathbb{C}^{n}$, Math. Z. 222 (1986), 407-424.

[Ra] T. Ransford, Potential Theory in the Complex Plane, Cambridge Univ. Press, 1995.

[Sa] A. Sadullaev, Plurisubharmonic measures and capacities on complex manifolds, Russian Math. Surveys 36 (1981), 61-119. 
[Si] J. Siciak, Extremal plurisubharmonic functions in $\mathbb{C}^{n}$, Ann. Polon. Math. 39 (1981), $175-211$.

Department of Mathematics

University of Education of Hanoi (Dai Hoc Su Pham Hanoi)

Cau Giay, Tu Liem, Hanoi, Vietnam

E-mail: ngquangdieu@hn.vnn.vn

Reçu par la Rédaction le 22.9.2002

Révisé le 21.2.2003 